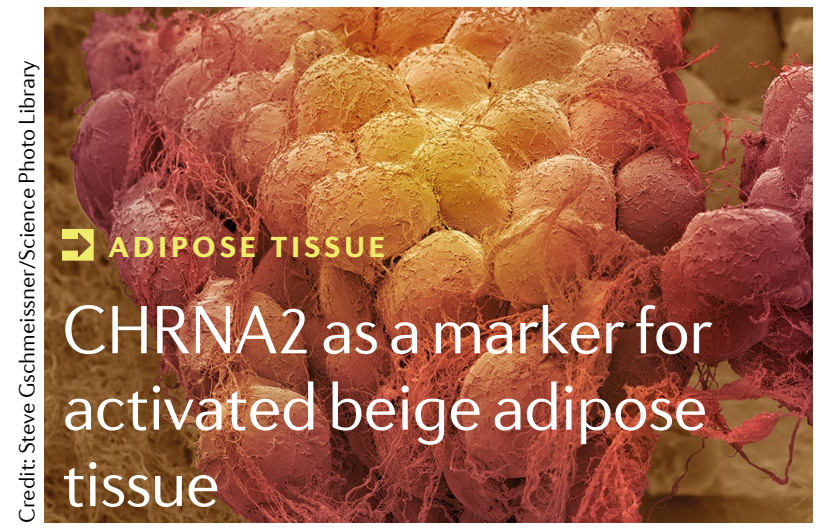

Beige fat cells are a potential therapeutic target for the prevention and treatment of human obesity, but little is known about the mechanisms underlying beige fat activation. Now, Jun Wu and colleagues have moved us one step closer towards this goal by showing that the $\alpha 2$ subunit of the nicotinic acetylcholine receptor (CHRNA2) is upregulated in beige fat cells following their activation.

"The therapeutic potential of beige fat cells is clear: genetic manipulations that create more beige fat in mice have strong anti-obesity and anti-diabetic effects and cumulative evidence supports a protective role of activated thermogenic fat in maintaining systemic metabolic homeostasis in humans," explains Wu. "In our study we used a calciumimaging assay in combination with Ucp1-CRE-RFP primary inguinal fat cell culture and flow cytometric analysis using ChAT-GFP reporter mice to investigate the molecular mechanisms and signalling networks that regulate beige fat development and activation."

The authors found that the expression of CHRNA2 is upregulated in activated beige fat cells from subcutaneous adipose depots. They demonstrated that that a subgroup of acetylcholine-producing $\mathrm{CD} 45^{+}$haematopoietic lineage cells within these depots mediate CHRNA2 signalling in beige adipocytes. Wu and colleagues then went on to investigate how this immune cell-beige adipocyte communication process is regulated during cold exposure and obesity.

"We found that Chrna2-knockout mice (in which the canonical $\beta$-adrenergic regulation of the beige fat remains intact) demonstrate a compromised adaptation to cold and exacerbated diet-induced obesity," adds Wu. "These results not only further confirm the physiological significance of beige adipocytes as an important metabolic regulator, but also highlight the essential role of catecholamine-independent, acetylcholine-CHRNA2 signalling in energy metabolism."

Wu and colleagues now want to investigate whether any of the acetylcholine-producing $\mathrm{CD} 45^{+}$cells within the subcutaneous fat tissue have a dominant role in regulating the activation of beige adipocytes that is mediated by CHRNA2. "The identification of such a subset would help to incorporate the acetylcholine-beige fat signalling pathway into the already identified signalling network between immune cells and adipocytes and place this newly defined pathway within the interconnected metabolic matrix," concludes Wu.

Alan Morris

ORIGINAL ARTICLE Jun, H. et al. An immune-beige adipocyte communication via nicotinic acetylcholine receptor signaling. Nat. Med. https://doi.org/10.1038/ s41591-018-0032-8 (2018)
NEUROENDOCRINOLOGY

\section{A novel pathway that controls feeding behaviour}

The brain regulates numerous behaviours that are fundamental to metabolism, including food intake and sleep; however, the precise brain-related mechanisms controlling these metabolic processes are unclear. The classic regulatory mechanisms include neuroendocrine control, whereby neuropeptides are released into the bloodstream, or synaptic communication between key brain regions. Now, Emily Noble, Scott Kanoski and colleagues describe a new signalling mechanism for melanin-concentrating hormone $(\mathrm{MCH})$, in which $\mathrm{MCH}$ is trafficked via the cerebrospinal fluid (CSF) to the neurons that activate feeding behaviour.

"In one of our previous papers we investigated neuropeptide systems that influence food intake. We noticed that the receptors for some peptides were in brain regions that were not innervated by the neurons that synthesized the peptide," explains Kanoski. Therefore, the authors hypothesized that the ventricular CSF, which is traditionally thought to provide mechanical protection for the brain, might be an active signalling pathway for some of these neuropeptides.

To investigate whether $\mathrm{MCH}$ is released into the CSF to increase food intake, the authors first determined the anatomical relationship of $\mathrm{MCH}$-producing neurons with the CSF using tract tracing and immunolabelling in a rat model. Noble reports that their investigation revealed that approximately one-third of all neurons that synthesize $\mathrm{MCH}$ communicate directly with the CSF.

Next, Noble and colleagues used chemogenetics to selectively activate $\mathrm{MCH}$-producing neurons that project into the CSF. "Not only did activating these $\mathrm{MCH}$ neurons increase the levels of $\mathrm{MCH}$ in the CSF, but it also caused the rats to eat more food," adds Noble. Finally,

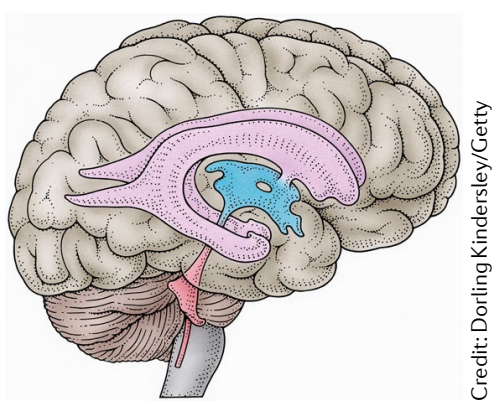

the group used a novel antibody trap to sequester endogenous $\mathrm{MCH}$ in the CSF, which prevented it from activating its receptor. The authors reported that following the antibody trap, the rats ate less food. Taken together, the findings reported by Noble, Kanoski and colleagues suggest that CSF transmission of $\mathrm{MCH}$ is important for normal food intake.

"Others have measured levels of neuropeptides, such as $\mathrm{MCH}$, orexin and oxytocin, in the CSF and correlated levels of these neuropeptides to various behaviours," explains Kanoski. "However, it was unclear whether peptides in the CSF are there because they are destined to be cleared from the brain as waste or whether they actually have some sort of biological role. Here, we reveal a biologically active role for neuropeptide transmission via the CSF"'

The authors now plan to determine whether similar CSF signalling cascades extend to other neuropeptides that influence food intake, such as orexin, oxytocin and glucagon-like peptide 1. "From a clinical perspective, knowing the endogenous mechanisms through which a brain-derived molecule acts in the brain to influence fundamental behaviours can potentially guide the [development of effective therapeutics]," concludes Kanoski.

Alan Morris

ORIGINAL ARTICLE Noble, E. E. et al. Control of feeding behavior by cerebral ventricular volume transmission of melanin-concentrating hormone. Cell Metab. https://doi.org/10.1016/j.cmet. 2018.05.001 (2018) 